\title{
Erratum to: Biomarkers of hepatic injury and function in neonatal hypoxic ischemic encephalopathy and with therapeutic hypothermia
}

\author{
Hemananda Muniraman ${ }^{1,2}$ • Danielle Gardner ${ }^{3}$. Jane Skinner ${ }^{4}$ - Anna Paweletz ${ }^{3}$. \\ Anitha Vayalakkad $^{5}$ - Ying Hui Chee ${ }^{6}$ - Clare Clifford ${ }^{5}$ - Sunil Sanka ${ }^{1}$. \\ Vidheya Venkatesh $^{6}$ - Anna Curley ${ }^{6,7}$ - Suresh Victor ${ }^{5,8}$ - Mark A. Turner ${ }^{3,9}$. \\ Paul Clarke ${ }^{1,4}$
}

Published online: 7 August 2017

(C) Springer-Verlag GmbH Germany 2017

\section{Erratum to: Eur J Pediatr}

DOI: $10.1007 / \mathbf{s 0 0 4 3 1 - 0 1 7 - 2 9 5 6 - 2}$

The original version of this article, unfortunately, contained errors.

The $p$ value columns in Tables 2 and 3 were inadvertently shifted up by one row during the copy editing process. Therefore all $p$ values as published in these tables did not

The online version of the original article can be found at http://dx.doi.org/ 10.1007/s00431-017-2956-2

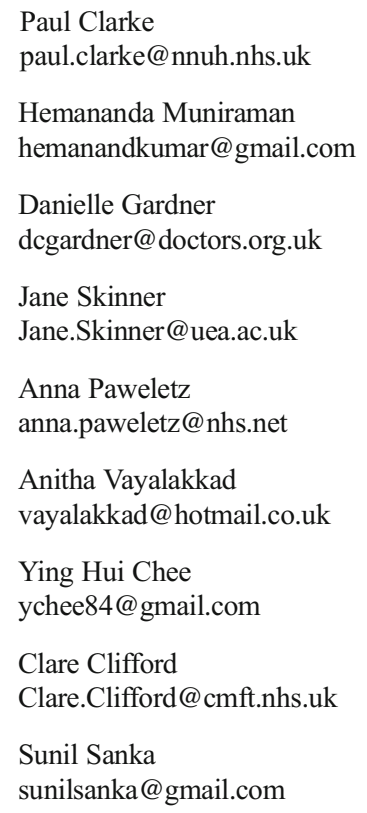

correspond to their correct data variables and thereby misrepresented the results of statistical analysis. The updated published version of this article has now corrected the $p$ value columns in Tables 2 and 3 such that all the previously misaligned $p$ values do now properly correspond to their correct respective rows of variables as were present in the authors' final submitted manuscript version.

The original article was corrected.
Vidheya Venkatesh

vidheya76@yahoo.co.in

Anna Curley

acurley@nmh.ie

Suresh Victor

suresh.victor@kcl.ac.uk

Mark A. Turner

Mark.Turner@liverpool.ac.uk

1 Neonatal Unit, Norfolk and Norwich University Hospitals NHS Foundation Trust, Norwich, UK

2 Division of Neonatology, Department of Pediatrics, Keck School of Medicine, Los Angeles County Medical Center, University of Southern California, Los Angeles, USA

3 Neonatal Unit, Liverpool Women's Hospital NHS Foundation Trust, Liverpool, UK

4 Norwich Medical School, University of East Anglia, Norwich, UK

5 Neonatal Unit, Central Manchester NHS Foundation Trust (St Mary's Hospital), Manchester, UK 
$6 \quad$ Neonatal Unit, Cambridge University NHS Foundation Trust (Addenbrooke's Hospital), Cambridge, UK

7 Neonatal Unit, National Maternity Hospital, Dublin, Ireland

8 Centre for Developing Brain, Division of Imaging Sciences and Biomedical Engineering, King's College London, King's Health Partners, St. Thomas' Hospital, London, UK

9 Department of Women's and Children's Health, Institute of Translational Medicine, University of Liverpool, Liverpool, UK 